\title{
La producción cafetera y su impacto en el crecimiento económico del departamento del Huila, Colombia*
}

\author{
Coffee production and its impact on economic growth \\ in the department of Huila (Colombia)
}

A produção de café e seu impacto no crescimento económico de Huila (Colômbia)

Recibido el 11 de agosto de 2016. Aceptado el 2 de febrero de 2017

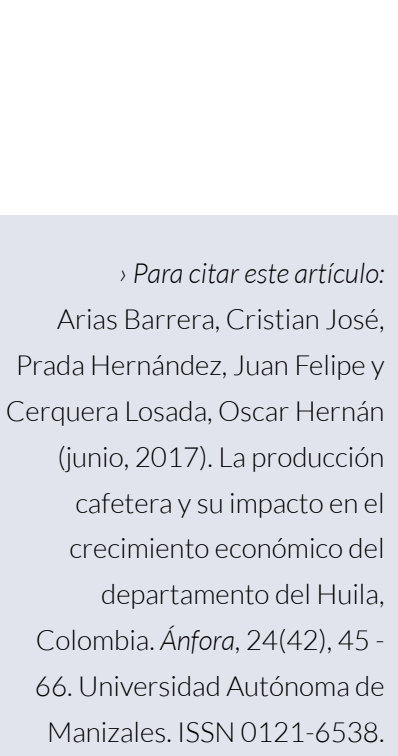

Cristian José Arias Barrera**

Colombia

Juan Felipe Prada Hernández***

Colombia

Oscar Hernán Cerquera Losada****

Colombia

\section{Resumen}

Objetivo: analizar los determinantes del crecimiento económico del departamento del Huila de 1980 a 2014, en particular del sector cafetero como uno de los principales aportantes. Metodología: con nivel de investigación explicativo y correlacional, se hizo análisis estadístico descriptivo y el desarrollo de un modelo

\footnotetext{
* Este artículo hace parte del proyecto denominado "Crisis Cafetera y el Impacto en el Crecimiento Económico en el Huila", aprobado y financiado por la Vicerrectoría de Investigación y Proyección Social de la Universidad Surcolombiana. Se realizó entre 13 de marzo de 2014 al 14 de marzo de 2015.

** Economista. Universidad Surcolombiana. Miembro del grupo de investigación IGUAQUE. Correo electrónico: cjab1@hotmail.com

*** Economista. Universidad Surcolombiana. Miembro del grupo de investigación IGUAQUE y CRE@. Correo electrónico: juanfeph@gmail.com

**** Magister en Economía de la Universidad Surcolombiana. Miebro del grupo de investigación IGUAQUE. Correo electrónico: oscar.cerquera@usco.edu.co
} 
econométrico de combinación de corte trasversal. Además, se usaron datos del sector cafetero y del Producto Interno Bruto para explicar las variaciones del PIB del Huila en relación con las fluctuaciones de las variables explicativas. Resultados: se encontró que el sector agrícola representa, en promedio, para el periodo evaluado, cerca del 17\% del PIB departamental. También se evidenció, que esta participación tiene una estrecha relación con los incrementos o decrementos del PIB cafetero. Conclusiones: se concluye la importancia del sector cafetero en la generación de crecimiento en la economía huilense, debido a la aplicación de tecnología y mejoramiento continuo del capital humano. Los sectores de la industria manufacturera, el comercio y la electricidad, no han tenido aportes significativos al crecimiento económico.

Palabras Claves: Crecimiento económico; Producción cafetera; Tecnología.

\section{Abstract}

Objective: the study aims to analyze the determinants of economic growth of the department of Huila from 1980 to 2014, mainly to analyze the coffee sector as one of the main contributors. Methodology: an explanatory and correlational research, a descriptive statistical analysis and development of an econometric model combination of crosscut was conducted. For this purpose, data from the coffee sector and gross domestic product were used to explain variations in GDP in the department of Huila in relation to the fluctuations of explanatory variables. Results: the findings revealed that the agricultural sector accounts for about $17 \%$ of the department GDP from the evaluated period. It also emerged that this contribution is closely related to increases or decreases in the coffee GDP. Conclusions: the importance of the coffee sector in generating growth in the economy in the department of Huila is due to the application of technology and continuous improvement of human capital. Manufacturing, trading and electricity sectors have not had a significant contribution to economic growth.

Keywords: economic growth; Production technology.

\section{Resumo}

Objetivo: analisar os determinantes do crescimento económico do departamento de Huila desde 1980 até 2014, em particular do setor cafeeiro como um dos principais contribuintes. Metodologia: nível de pesquisa explicativa e de correlação, fez-se análise estatística descritiva e o desenvolvimento de um modelo econométrico de combinação de corte transversal. Para fazer isso, foram usados os dados do setor cafeeiro e do 
Produto Interno Bruto para explicar as variações do PIB de Huila em relação às flutuações das variáveis explicativas. Resultados: achou-se que o setor agrícola representa, em média, para o período avaliado, cerca do 17\% do PIB departamental. Evidenciou-se também, que essa participação está intimamente relacionada com os aumentos ou diminuições do PIB cafeeiro. Conclusões: a importância do setor cafeeiro na geração de crescimento na economia huilense é devido à aplicação de tecnologia e à melhoria contínua do capital humano. Os setores da indústria manufatureira, o comércio e a energia elétrica, não tiveram uma contribuição significativa para o crescimento económico.

Palavras-chave: Crescimento econômico; Tecnologia de produção. 


\section{Introducción}

En las últimas décadas, algunas economías mundiales presentaron un comportamiento inestable en las estructuras macroeconómicas; ello muestra una correspondencia positiva entre crecimiento económico y la razón de inversión a producto, apoyada por los efectos del progreso técnico y una relación negativa respecto a la tasa de interés asociada al costo de oportunidad (Ross y David, 1992).

Ahora, los costos de oportunidad de los componentes de los productos producidos por una economía tienden a ser volátiles, pero, en cierto punto, mantienen una evolución relativamente constante en el crecimiento económico. De este modo, estas variables se diseñan en función del producto agregado que se modifique con el tiempo, teniendo en cuenta los cambios, en otros factores, en las características estructurales del proceso de crecimiento (Naciones Unidas [NU]; Comisión Economica Para América Latina y el Caribe Subsede México [CEPAL], 2003).

Tales cambios se deben aplicar de forma transversal en cada uno de los sectores que se complementan; esto, con el fin de procurar fortalecimiento en los agregados económicos que generen un desarrollo económico equilibrado e incentiven el bienestar social. Se trata de hallar condiciones que puedan, en gran medida, sostener los niveles productivos evolucionados a través de la producción del conocimiento, que debe estar al alcance de todos. Lograrlo, requiere una búsqueda de alternativas que produzcan cambios sustanciales y, para ello, es indispensable la aplicación del conocimiento (Clark, 1923), porque es éste el único instrumento de producción que no está sujeto a la disminución de los ingresos.

Como lo sugieren los modelos de crecimiento endógeno, existen diversas formas de convergencia económica (Galor, 1996), que otorgan importancia al Capital Humano. Los modelos de Romer (1986), Lucas (1988) y Barro (1991) muestran que el Capital Humano, es el grado de calidad y de formación de las personas vinculadas a los procesos productivos y que generan convergencias hacia un mayor crecimiento económico en el largo plazo. Es por ello que el conocimiento en las respectivas unidades productivas, es un factor acumulable para el crecimiento económico y que se ajusta a los requerimientos del entorno económico. 


\section{Producción cafetera del Huila ${ }^{1}$}

Del sector agrícola huilense hace parte el café que aportó, en el año 2013, un 6.6\% (Departamento Administrativo Nacional de Estadísticas [DANE], 2013) al crecimiento económico del departamento. El café es uno de los productos más competitivos en la economía internacional y su influencia es indiscutible en el desarrollo económico. En el departamento del Huila, tiene un fuerte impacto en el total las exportaciones, pero no en el desempeño del mercado global, ni siquiera en el de Colombia, aunque se haya demostrado una productividad con alto valor agregado como el café especial (Bolsa Mercantil de Colombia [BMC], 2011).

La variabilidad económica ha hecho que el sector cafetero no sea estable, principalmente por los precios internacionales, la coyuntura económica -que afecta el mercado interno (consumo, precios internos, distribución, etc.)- y las variaciones del medio ambiente, que permiten que el comportamiento del sector sea aún más variable en el tiempo.

La poca producción ha sido una de las consecuencias del decrecimiento del sector cafetero a causa de la falta de cohesión entre el medio ambiente y la tecnología aplicada en la producción (Ampié, 2000), conllevando a un bajo ingreso de los caficultores y la pérdida de empleos directos, indirectos, formales o informales, sobrecostos en los insumos en los momentos de daños en los cultivos.

Son estos factores los que han conducido a la variabilidad de la producción cafetera, caracterizada por la baja producción que conduce al desempleo y la reducción de las exportaciones drásticas como se han experimentado en años anteriores (Kalmanovitz y López, 2006). Todo ello lleva a cavilar que esta actividad productiva se ejerce en medio de notables dificultades, riesgos e incertidumbres.

En ese sentido, surge el interrogante ¿Por qué el sector cafetero es determinante en el crecimiento de la economía huilense en el período 1980 a 2014 ?

El objetivo de este artículo, entonces, es dar a conocer las medidas que el gobierno colombiano ha tomado para el sector cafetero, los factores influyentes

1 El departamento del Huila es uno de los 32 departamentos de Colombia que está localizado al suroccidente del país en la región andina, su capital es Neiva. El Huila últimamente ha sido reconocido a nivel mundial por su producción de Café de alta calidad. 
del sector cafetero y de los agregados económicos que determinan el crecimiento económico del Huila. Además, a partir de la caracterización de la base de datos del periodo 1980 a 2014 se desarrolla un modelo econométrico de corte transversal. Finalmente, se pretende que el desarrollo de esta investigación aporta conocimiento para los caficultores y organizaciones vinculadas permanentemente con el desarrollo de la economía del Huila.

\section{Metodología}

Se desarrolló un estudio mediante el método inductivo bajo los criterios de un enfoque de tipo explicativo y correlacional. Se tomó como fuente la información estadística de los libros oficiales de la Secretaria de agricultura y de Planeación Departamental del Huila, del DANE; además se consultaron artículos de revistas indexadas, informes, libros en versión digital e impresa.

Su análisis se realizó en dos partes: la primera, tomó como referencia el esquema por etapas para llevar el análisis documental: la búsqueda de los documentos existentes y disponibles, la clasificación y selección de los documentos identificados para su respectivo análisis. En esta primera parte, también se apoyó con trabajo de campo mediante entrevista conversacional con los principales actores de la investigación (Campesinos caficultores), de los municipios de Garzón y Acevedo ubicados en el centro y sur del departamento del Huila.

En el desarrollo de la segunda parte se usaron datos de los sectores macroeconómicos del Huila, especialmente del sector cafetero; se aplicaron las variables área sembrada $(\mathrm{Ha})$, área cosechada $(\mathrm{Ha})$, rendimiento $(\mathrm{Kg} / \mathrm{Ha})$, producción (Ton), precio interno base y el PIB Cafetero, con los agregados de la economía huilense de los sectores de construcción, minería, comercio, industria manufacturera y electricidad, aplicando estadísticas descriptivas para el análisis de cada una de estas variables, así como la relación entre cada una de ellas.

Para lo anterior, se realizó, en primera instancia, la estimación de los datos en términos de tasa de crecimiento con el fin de realizar el análisis estadístico descriptivo y su tipo de correlación (Correlación de Pearson) de cada uno de los datos. De esta manera, se implementó el modelo econométrico de combinación de corte trasversal, el cual fue ajustado a un diseño de investigación no 
experimental o ex-post-facto ${ }^{2}$, como herramienta para estimar la causalidad entre el crecimiento económico del Departamento del Huila con la inferencia de las variables macros elegidas. Se utilizó como metodología el enfoque de estudio de Galindo y Catalán (2003) y teniendo en cuenta el estudio de Calzada (2002).

Para el cálculo del sector cafetero fueron las variables de producción cafetera y el valor promedio de carga, el producto entre estas dos variables es el PIB cafetero del Departamento, que luego fueron restadas al PIB del sector Agropecuario. Todos los datos se deflactaron con base en el año 2007 (Lora, 2008) y se pasaron a tasas de crecimiento, para el modelo econométrico.

Con todas las variables en una misma unidad de medida se aplicaron estadísticas descriptivas para el análisis de cada una de ellas y su relación con las demás. Luego, se analizó el grado de asociación entre las variables por medio de la correlación de Pearson, para aplicar el modelo econométrico, de acuerdo con la información empírica y teórica (Spanos, 1986), cumpliendo con los supuestos econométricos (Wooldridge, 2010).

\section{Resultados}

Los resultados se presentan en tres apartados. El primero se relaciona con los aspectos más comunes que se extrajeron de las entrevistas conversacionales. El segundo apartado hace relación al análisis estadístico descriptivo del comportamiento de la actividad cafetera en el departamento del Huila. El último apartado, presenta los resultados del modelo econométrico de combinación de corte transversal.

\section{Gráfico 1. Diagrama causal}

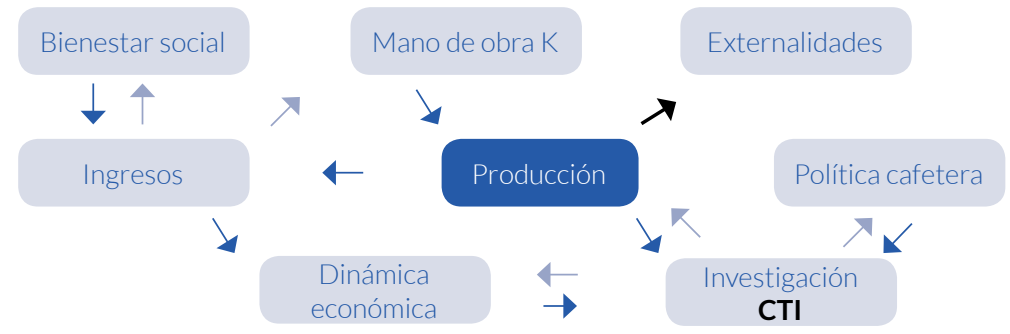

Fuente: elaboración propia

2 Expresión que significa "después de hecho", haciendo alusión a que primero se produce el hecho y después se analizan las posibles causas y consecuencias, por lo que se trata de un tipo de investigación en donde no se modifica el fenómeno o situación objeto de análisis (Bernardo y Caldero, 2000). 
El gráfico destaca la importancia del sector cafetero para la generación del crecimiento económico en el departamento del Huila. Y para que sea aún mayor ese aporte se deben tener en cuenta todas las condiciones favorables y desfavorables que existen en torno al sector; de esta manera se tendrá claro qué lo afecta o favorece, para tomar decisiones acertadas que ayuden fortalecer la caficultura huilense.

Es importante la aplicación de Ciencia, Tecnología e Innovación para generar una mayor productividad y facilidad en la obtención de resultados. Para ello, contribuyen las políticas del gobierno nacional y la Federación Departamental de Caficultores del Huila, de acuerdo con la dinámica de la economía que debe impulsar estos procesos.

Para la recolección del fruto y según la extensión de tierra cultivada, se demanda mano de obra que es remunerada con base en las condiciones del mercado o determinación del caficultor. Los ingresos por mano de obra, mejoran la calidad de vida y son devueltos al mercado para incentivar dinamismo al sector cafetero y a la economía en general. También inciden en el proceso de recolección algunos factores externos que impiden en algunas ocasiones una mejor productividad, como los repentinos cambios climáticos, paros agrarios, la volatilidad del dólar, etc.

Para identificar las condiciones de los caficultores y los procesos productivos, se visitaron seis fincas cafeteras, tres de ellas ubicadas en el municipio de Garzón y otras tres en el municipio de Acevedo Huila, teniendo el apoyo y acompañamiento directo del Comité Departamental de Caficultores del Huila.

En gran parte, la caficultura se ha trasmitido de generación a generación; la mayoría de quienes ejercen esta actividad son hombres, pero ha sido tradicional el aporte de las mujeres. A esta actividad contribuye el Comité Departamental de Cafeteros con incentivos que aumentan la productividad; por ejemplo, los servicios de extensión que brindan asistencia técnica para nuevos estudios para el control de plagas, de buenas prácticas ambientales, del buen manejo de fertilizantes, entre otros. La mayor parte de los caficultores han emprendido ese camino para lograr los mejores beneficios.

El departamento, en materia de agroindustria, ha priorizado sectores comunes como el mejoramiento genético cafés especiales, frutas tropicales con el objeto de lograr procesos de valor agregado con componentes de ciencia, tecnología e innovación. Proyectos necesarios para el desarrollo económico y 
social, así mismo el desarrollo y la creación de una zona franca (BIOCAFÉ). Este proyecto de carácter agroindustrial, liderado por el municipio de Pitalito y los caficultores del sur del departamento del Huila, cuenta con la mayor producción de café tecnificado de la región. Con una inversión inicial de 7.288 millones de pesos, para un área de 10.300 metros cuadrados, que permite la construcción de 9 zonas específicas destinadas a las actividades de ensamblaje de maquinaria agrícola, producción y comercialización de fertilizantes y fungicidas líquidos, transformación en la producción de café, entre otros.

Otra de las consideraciones sobre los resultados de este estudio, es que la ayuda que recibe el sector agropecuario debe ser en herramientas y especies. Los subsidios en efectivo tienden a desviarse de la inversión en la productividad cafetera.

La participación de algunos caficultores en concursos de tasa de excelencia, han despertado la necesidad de otros de llevar un mejor producto al mercado, pero hace falta un poco más. Por ejemplo, que asociaciones incentiven interacción entre los caficultores y no caficultores de las regiones para que, unidos, se genere bienestar para todos. Además, se debe estimular sentido de pertenencia no sólo de los caficultores, sino también de los consumidores locales, que compran productos de café de empresas que no son del Huila y que no presentan las altas tasas de calidad que en estas tierras se produce.

Uno de los mayores inconvenientes que se ha presentado el sector es, sin lugar a dudas, el paro del año 2013. Todos los inconvenientes que ocasionaron los paros campesinos, para unos no fueron de utilidad, porque hoy día no se han hecho efectivos en totalidad los acuerdos a que se llegaron. Además, la fecha de inicio del paro fue la misma en la que tenían que recolectar el fruto; la mano de obra escaseó y la pérdida fue históricamente cuantiosa.

Ahora bien, las políticas son importantes, por ejemplo, las cédulas cafeteras, que sirven como identificación del caficultor quien recibe, por medio de ésta, los apoyos del gobierno, de la Federación o el Comité en los programas que ofrecen; con esta identificación también se puede participar en las elecciones cafeteras, recibir el pago de sus cosechas, entre muchos otros servicios. Otra política que se destaca para el departamento es la adoptada desde el 2008 por la Federación Nacional de Cafeteros, con la renovación de cafetales que eran susceptibles a las plagas y cambios climáticos; esto, para tener una mayor productividad acorde con el medio ambiente: en el Huila, gran parte de los procesos se da por fermentación natural y secado al sol y se destacan las variedades de café Colombia, Castilla y Caturra. 
Los caficultores que participaron en este estudio expresaron que una de las dificultades es la poca importancia de la economía rural por parte del Estado. Esto dificulta el desarrollo del campo, que necesita mejores vías de acceso que permitan la entrada y salida de productos a otros países, con el fin de aumentar competitividad y, por ende, mejor calidad de vida para los caficultores.

\section{Comportamiento de la actividad cafetera en el Departamento del Huila.}

\section{Gráfica 2. Área sembrada y Área cosechada. Ha. del cultivo del café. 2007-2013}

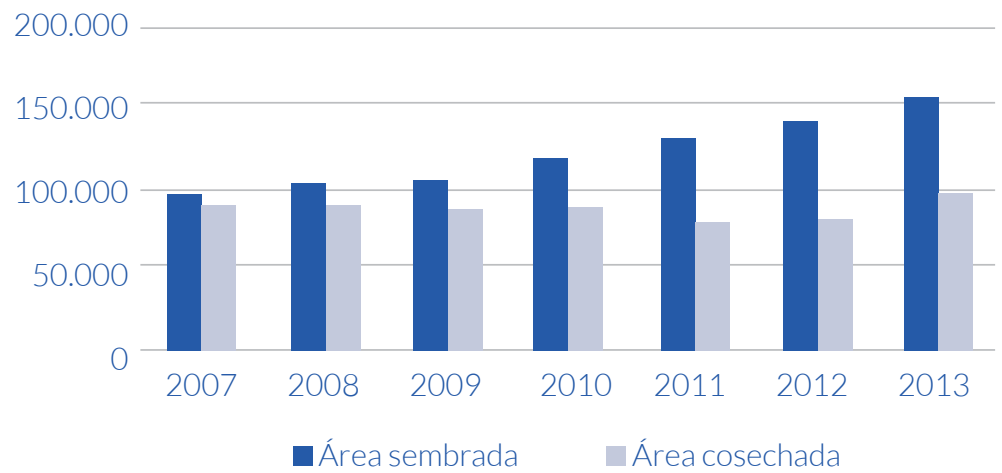

Fuente: elaboración propia con base

a Información de la Secretaría de Agricultura y Minería. Gobernación del Huila.

Teniendo en cuenta que la actividad cafetera es de gran importancia en la economía huilense, fue imprescindible analizar su comportamiento durante los últimos años. En cuanto al área sembrada el crecimiento promedio ha sido de 7,2\% durante el periodo 2007-2013; en el último año creció en 10,5\%, es decir se sembraron 14.677 hectáreas más. El área cosechada ha crecido a una tasa promedio de $1,9 \%$ en el periodo analizado, aunque durante el año 2013 creció un 21\%, es decir aumentaron 16.796 hectáreas cosechadas frente al año 2012. Esta situación puede explicarse por el inicio de producción de las hectáreas sembrada en años anteriores.

En relación con el área sembrada frente al área cosechada en el departamento del Huila, el coeficiente en el año 2013 fue de 63\%; es decir, de cada 100 hectáreas sembradas se cosechan 63 hectáreas. Esta relación ha disminuido en el tiempo; por ejemplo, en 2007 y 2008 el coeficiente era de 93\%, esto puede explicarse por 
el aumento de las hectáreas sembradas como lo muestra el grafico 2 y el bajo crecimiento del área cosechada.

\section{Producción y rendimientos}

\section{Gráfica 3. Producción de café en el Huila 1980-2012.}

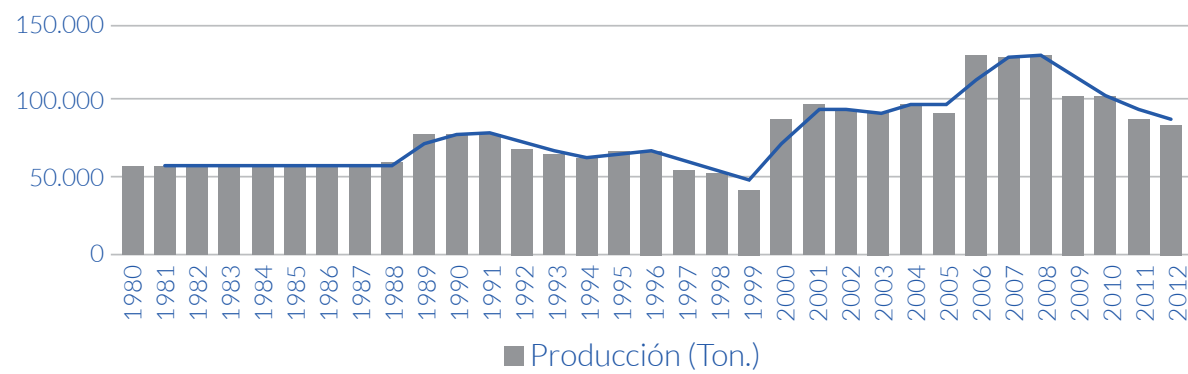

Fuente: elaboración propia con base en información de la Secretaría de Agricultura y Minería. Observatorio de Territorios Rurales.

La producción, durante el periodo 2007-2013, ha tenido una tasa de crecimiento promedio anual de $0,5 \%$, aunque durante el año 2013 creció en un $35 \%$ respecto al 2012; es decir, la producción aumentó en 29.904 toneladas. Es por eso que durante este año, 2012, como dice el informe de coyuntura económica de la Cámara de Comercio de Neiva, la producción agrícola, especialmente la producción de café, contribuyó en buena medida a mejorar la dinámica de la economía del departamento, a pesar de los paros protagonizados por sus inconformidades en cuanto a los precios y baja rentabilidad del cultivo.

La productividad del cultivo del café ha disminuido en el tiempo como lo muestra el gráfico 4, mientras en el año 2008 el rendimiento por hectárea era de 1,5 toneladas, en el año 2011 y 2012 tan solo fue de 1,1 Ton/Ha, aunque en el año 2013 aumentó a 1,2 la productividad en el departamento siendo aún es baja. Como se evidenció, las consecuencias dadas a causa de la apertura económica en los años 90s seguida con los fenómenos climáticos o factores exógenos que altera el nivel de producción de los cultivos generó un bajo rendimiento en la productividad del cultivo por hectárea. 


\section{Relación costos y precio del café}

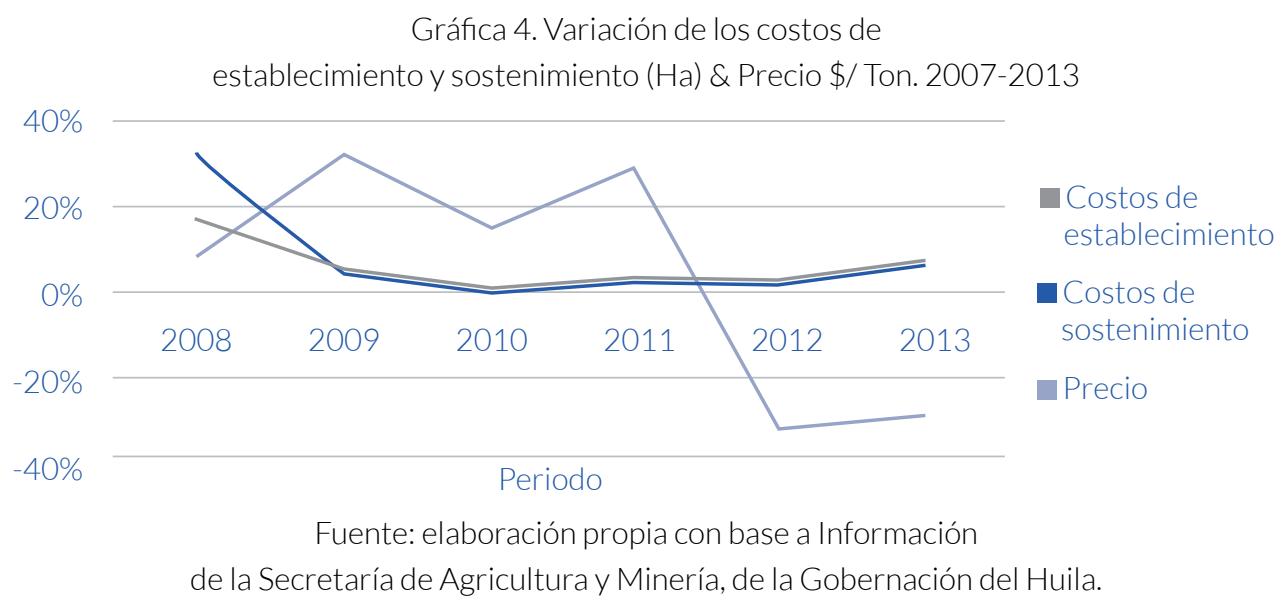

Una de las principales problemáticas, según los productores del grano, en las pasadas manifestaciones y paros campesinos (2011-2013) ha sido el aumento de los costos de producción, especialmente por el alza de los precios de los fertilizantes. La grafica 4 muestra los costos de establecimiento de una hectárea de café y del sostenimiento de la misma. Los costos de establecimiento han aumentado a una tasa promedio anual de $6 \%$ durante el periodo analizado y los costos de sostenimiento en $8 \%$, por encima de la tasa de inflación promedio registrada durante estos años que fue de 4,35\% según el Banco de la República.

En cuanto al precio del café, se observa en la gráfica 4 una variación promedio anual de 4\%, con una tendencia creciente durante el periodo 2007-2011; sin embargo disminuye a tasas significativas entre los años 2012 y 2013. Así, el comportamiento, en conjunto, de los costos de establecimiento y sostenimiento frente al precio por tonelada (Grafica 4) evidencia las disparidades en las tendencias, pues aunque en el periodo 2008-2011 se mantenían crecientes las variaciones de los costos y el precio, a su vez el crecimiento del precio estaba por encima de los costos; mientras que durante los años 2012 y 2013 los costos mantenían tasas crecientes frente a los precios que se desplomaron en tasas significativas; esta situación se reflejó en las grandes manifestaciones de inicios de esta década en la que los productores manifestaron la problemática social del campo, puesto que el precio del café no era sustentable frente a los costos.

El análisis por sectores económicos permite identificar la relación de las variables que determina el crecimiento económico del departamento, estableciendo las características generales de cada una. En la década de los 80 s 
en la rama de actividad del sector agropecuario sin cafés representó un $24 \%$, dentro de este sector no se tuvo en cuenta la variable café como tal, por tanto, ésta presentó una participación en el PIB departamental del 39\%.

Ya para los 90s el sector agropecuario presentó una disminución en cuatro puntos porcentuales (4\%), teniendo un $24 \%$ y un $20 \%$ para los años 80 s y 90 s, respectivamente. Para esta década se presentó una baja participación también del sector cafetero, el cual fue de un $23 \%$.

Antes de los 90s se tenía una política proteccionista que dómino el intercambio comercial con otros países durante varios años. Teniendo como consecuencia a este tipo de política sobre producción de los productos nacionales logrando un alza de los precios, pero con una poca demanda. Las fuertes críticas contra la apertura de los 90s se basan, generalmente, en análisis parciales de la correlación positiva entre la caída del arancel promedio y el desempeño de la producción agrícola. Además, un análisis más completo debe incorporar el hecho de que, junto con la apertura, confluyeron hechos adicionales como un "niño" climático, particularmente intenso en 1992, y una caída fuerte de los precios internacionales de los productos agrícolas durante la mayor parte de la década, la revaluación del peso y la expansión de los cultivos ilícitos (Kalmanovitz y López, 2006).

Para los 2000s, la mayor actividad económica representativa para el Departamento, sigue siendo el agropecuario sin incluir café con $16 \%$ de participación; bajo cuatro puntos (4\%) con respecto a la década de los $90 \mathrm{~s}$, pero si se revisa con respecto a la de los 80 s, se presenta una disminución de un ocho por ciento ( $8 \%$ ), debido a que venía desde los 90 s sufriendo una caída en la producción agropecuaria del Departamento; sin embargo, a mitad de la década se fue recuperando gradualmente, aunque al final volvieron fenómenos climáticos (niña) y coyunturales en el Departamento.

Para los últimos años de estudio de esta investigación 2010-2012, 14\% fue la participación del sector agropecuario, porcentaje significativo para la década en sus primeros tres años, pero no tanto para la serie de tiempo, ya que su participación es de un $19 \%$ del total. La participación para el café fue de $18 \%$, con una producción de 104337, 88283 y 85213 para 2010, 2011 y 2012, respectivamente; se presentó, entonces, una reducción de 16054 Ton., es decir un $-15,39 \%$ de la producción para el 2011 con respecto al 2010, causado

3 Llámese al sector agropecuaria (sin café) al conjunto de actividades agrícolas, producción pecuaria y caza, silvicultura, extracción de madera y actividades conexas, pesca, producción de peces en criaderos y granjas piscícolas; actividades de servicios relacionadas. 
principalmente por el fenómeno de la niña y la reducción en el precio en el mercado. Dicho lo anterior, no se tuvo en cuenta para la calificación, ya que para el Departamento del Huila en el año 2011 fue declarado como primer productor del país y en calidad, superando históricamente al eje cafetero, partiendo de la renovación de cafetales.

Seguido del sector agropecuario está la minería; entiéndase esta variable como explotación de mina y canteras; la segunda activad económica importante del Departamento para los 80s, con una participación del 21\%. En los 90s, la minería presentó una disminución de cuatro puntos porcentuales (4\%), es decir que para esta misma década la minería contribuyó al PIB Departamental en un $17 \%$, frente a la década de 2000 s que presentó un aumento significativo en esta actividad económica para el crecimiento del PIB en dos puntos porcentuales (2\%); ello es significativo pues durante los últimos 13 años ${ }^{4}$ ha mantenido un promedio de los dos puntos porcentuales, esto quiere decir que ha conservado la producción de la explotación de mina y canteras.

En relación con el proceso de industrialización, la industria manufacturera en el Departamento del Huila no es un renglón tan significativo para el PIB, de acuerdo con los testimonios de los entrevistados en este trabajo. Casi siempre se han referido a este sector de esta forma, no por su importancia para el desarrollo del sector secundario como actividad económica sino, por su aporte al crecimiento de la región. En la serie de datos estudiados en esta investigación representa el $5 \%$ respecto al PIB total. Para los 80 s representó siete puntos porcentuales $(7 \%)$ para el PIB de esos 10 años; en los 90s registró cinco puntos porcentuales (5\%) presentando una caída en la producción de dos puntos (2\%). El 19\% fue lo que se obtuvo como participación en los 2000 s con un incremento significativo en 14\% respecto a la anterior década.

En los 80s, el sector electricidad ${ }^{5}$ representaba dos puntos porcentuales $(2 \%)$ y para la década de los 90 s se obtiene un incremento en cinco puntos porcentuales (5\%), es decir, que para esta década el sector de electricidad, gas y agua representó un siete por ciento (7\%) del PIB Departamental del mismo año.

La siguiente década (2000s) registró una actividad negativa de menos dos puntos porcentuales (-2\%), al representar un cuatro por ciento (4\%) al PIB. Para

\footnotetext{
4 Contando los tres primeros años de la década de 2010.

5 Electricidad, gas y agua: Empresa dedicada a la producción y/o distribución de energía en la forma de electricidad gas o agua.
} 
el primer trimestre de la década de 2010, esta actividad económica siguió con la misma participación.

Esta investigación también arrojó que para la década de los 80 s la construcción presentó una participación de siete puntos porcentuales $(7 \%)$, con un leve aumento de un punto porcentual (1\%) para la década de los $90 \mathrm{~s}$ y dos puntos $(2 \%)$ para la de 2000s. Para los últimos siete años se evidenció un aumento de siete por ciento (7\%), es decir que para el último periodo de la serie se obtuvo $15 \%$ del sector construcción ${ }^{6}$ y obras civiles como aporte al crecimiento económico de la economía huilense.

Al analizar el comportamiento de esta variable, mediante la participación con respecto al PIB del Huila, se demostró que en la década de los 80 s el comercio ${ }^{7}$ participó con nueve puntos porcentuales $(9 \%)$, para la década siguiente fue de ocho puntos $(8 \%)$, en los 2000 s siguió su participación, con una reducción de menos dos puntos $(-2 \%)$ y para los últimos tres años analizados siguió a la baja con una participación de cinto por ciento $(5 \%)$.

El sector transporte para el Departamento del Huila, no es tan significativo por oscilar entre las tres décadas y el primer trimestre de la del 2010 s en un punto porcentual (1\%): para los 80 s nueve puntos $(9 \%)$ porcentuales, ocho $(8 \%)$ por ciento para los 90 s, nueve por ciento ( $9 \%$ ) y ocho por ciento para los $2000 \mathrm{y}$ primer trimestre de los 2010 , respectivamente.

\footnotetext{
6 Según el Centro Andino de Altos Estudios, se refiere a la edificación de una obra de ingeniería, arquitectura o albañilería (Centro Andino de Altos Estudios [CANDANE], 2007).

7 El comercio es la compra y venta al por mayor y al por menor de mercancías (bienes que van al mercado), nuevas y usadas y que no son sometidas a transformaciones en su naturaleza intrínseca durante las actividades inherentes a la comercialización, transporte, almacenaje, empaque, re empaque, embalaje, etc., o en aquellas a adecuar, exhibir, presentar o promocionar las mercancías objeto de la venta.
} 


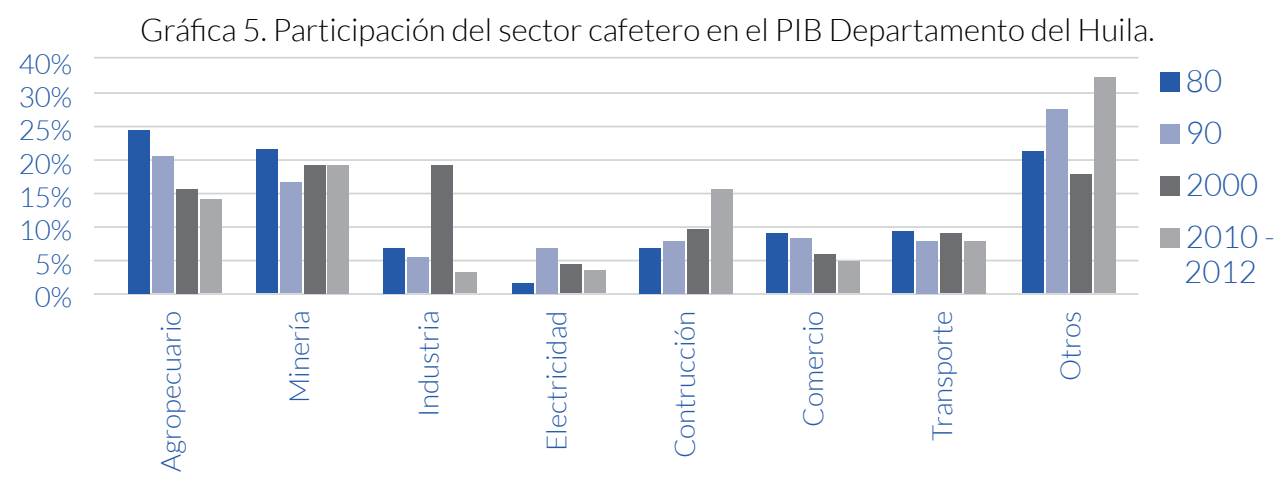

Fuente: elaboración propia con información de la Secretaría de Agricultura y Minería.

Observatorio de Territorios Rurales. Evaluaciones Agropecuarias Municipales.

La siguiente sección es el resultado del modelo econométrico de corte transversal que analiza los determinantes de crecimiento del departamento a nivel macroeconómico:

Se buscó crear un modelo econométrico acorde con la información empírica y teórica (Spanos, 1986) y que explique la relación de las variables respecto al crecimiento económico (PIB) del departamento del Huila, mediante un modelo de combinación de corte transversal (Wooldridge, 2010), como herramienta para estimar la causalidad entre el crecimiento económico del departamento del Huila y las variables económicas elegidas. El modelo de regresión con una variable y variables explicativas $X_{1}, X_{2}, X_{3}, X_{4}, \ldots, k_{n}$, el modelo se puede escribir de la siguiente forma:

$$
Y=\beta_{o}+\beta_{1 \times 1}+\beta_{2 \times 2}+\beta_{3 x 3}+\beta_{n \times n}+u_{n} \quad n=1,2,3 \ldots \mathrm{N}
$$

Donde $\beta_{0}$ es el intercepto, $\beta_{1}$ mide el cambio en respecto a $X_{1}$, manteniendo constantes todos los demás factores, $\beta_{2}$ mide el cambio en y respecto a $X_{2}$, conservando constantes todos los demás factores, etc. La variable $u$, es el termino de error o perturbación y contiene otros factores que no se incluyeron en el modelo, pero que allí se recogen de forma colectiva.

El modelo final se expresa con la siguiente ecuación:

$$
\begin{gathered}
\text { PIB }_{t}=\beta_{0}+\alpha_{1}\left(\text { pibCAFE } E_{t}\right)+\beta_{1}\left(\text { AGROSCAFE }_{t}\right)+\beta_{2}\left(\text { TRANS }_{t}\right)+\beta_{3}\left(\text { CONST }_{t}\right)+\beta_{4}\left(\text { MIN }_{t}\right) \\
+\beta_{5}\left(\text { COM }_{t}\right)+\beta_{6}\left(\text { INDM }_{t}\right)+\beta_{7}\left(\text { ELECT }_{t}\right)+\mu \mathrm{t}
\end{gathered}
$$


Los $\beta$ corresponden a los parámetros de los agregados económicos del departamento del Huila y $\alpha$ para la variable del sector cafetero. El sector comercio, la industria manufacturera y la electricidad no resultaron estadísticamente significativas en el modelo. La siguiente tabla muestra la incidencia de los distintos factores en el crecimiento de la economía Huilense.

Tabla 1. Determinantes del Crecimiento en la Economía del Departamento del Huila.

\begin{tabular}{|c|c|}
\hline PIB & Coeficiente \\
\hline Variables Observadas & \\
\hline \multicolumn{2}{|l|}{ Sector Cafetero } \\
\hline PIB Café & $\begin{array}{c}0.0844^{* * *} \\
(2.54)\end{array}$ \\
\hline PIB Agropecuario sin café & $\begin{array}{c}0.2487^{* * *} \\
(2.94)\end{array}$ \\
\hline Transporte & $\begin{array}{c}0.1186^{* * *} \\
(3.29)\end{array}$ \\
\hline Construcción & $\begin{array}{c}0.0587^{* * *} \\
(5.70)\end{array}$ \\
\hline Minería & $\begin{array}{c}0.1102^{* * *} \\
(5.05)\end{array}$ \\
\hline R Cuadrada & 0.8249 \\
\hline
\end{tabular}

Fuente: Elaboración Propia

$$
\begin{aligned}
& \text { PIB }_{t}=\beta_{0}+\alpha_{1}\left(\text { pibCAFE }_{t}\right)+\beta_{1}\left(\text { AGROSCAFE }_{t}\right)+\beta_{2}\left(\text { TRANS }_{t}\right)+\beta_{3}\left(\text { CONST }_{t}\right)+\beta_{1}\left(\text { MIN }_{t}\right) \\
& +\beta_{5}\left(\operatorname{COM}_{t}\right)+\beta_{6}\left(I N D M_{t}\right)+\beta_{7}\left(E L E C T_{t}\right)+\mu \mathrm{t}
\end{aligned}
$$

Se evidencia que el sector cafetero es importante para el crecimiento del departamento del Huila. En la década de los 80 s el año que mayor crecimiento tuvo fue el de 1985, en el que el PIB del sector creció 52.48\% respecto al año anterior. En la década de los 90 s, el año más de crecimiento fue 1995 cuando se obtuvo un crecimiento de $29.32 \%$ respeto al año de 1994 .

En esta década, se presentan algunos problemas que no permitían el fortalecimiento de la caficultura departamental. Para el año 2000, se evidencia el mayor crecimiento del sector cafetero con un $92.95 \%$ respecto al año de 1999 , 
tomando más fuerza como sector, con la adopción de políticas desde la Federación Nacional de Cafeteros y el Comité Departamental de Cafeteros. Una de las políticas que ayudaron a fortalecer el sector fue la renovación de cafetales viejos, improductivos y susceptibles a las plagas, a unos más resistentes y productivos.

En el periodo de este análisis, el sector agropecuario sin café, mostró siempre tasas de crecimiento importantes, siendo líder en el territorio nacional, según la Gobernación del Huila (2014). Este sector es el más significativo dentro de los sectores económicos y lo evidencia el modelo econométrico. Si el sector cafetero crece en $1 \%$, se generará un crecimiento $0.0844 \%$ en la economía departamental, manteniendo constantes las demás variables.

El sector de la construcción empieza a tener mayor importancia en el año 2006, dinámica que surge desde el desarrollo de políticas gubernamentales que se empiezan a adoptar, con la generación de viviendas nuevas, obras públicas y privadas que están en pro del bienestar social. Este sector tiene un efecto positivo en el mercado laboral, ya que acapara un moderado porcentaje de la fuerza de trabajo, especialmente la no calificada. Con el crecimiento del 1\% de este sector, se generará un crecimiento de 0.0587\% en el PIB del departamento del Huila.

El Sector minero, tiene un gran potencial que, al ser bien aprovechado con buenas prácticas ambientales, puede contribuir al crecimiento económico de la región. Los recursos mineros del Huila están clasificados en materiales de construcción, materiales de uso industrial y metales preciosos (Fajardo Palencia, 2013). La variable resulta estadísticamente significativa con una probabilidad de $1 \%$. Con el crecimiento del $1 \%$ de esta actividad, habrá un crecimiento económico de $0.1102 \%$.

\section{Conclusiones}

Es innegable la importancia del sector cafetero en la economía internacional; en el departamento del Huila inicia aproximadamente a inicios de siglo XXI. En general, el comportamiento de la economía cafetera ha sido históricamente variable y ello justifica un análisis esmerado de lo que ocurre en el contexto departamental. Sin dejar a un lado los sectores macroeconómicos que conforma la economía huilense. Los resultados de este estudio son consistentes en demostrar la importancia del capital humano y su aporte al crecimiento económico. 
Desde un enfoque de la teoría del crecimiento endógeno, en el presente trabajo se aplicó un modelo de combinación de corte trasversal. Los resultados de la metodología aplicada, muestran la importancia del sector cafetero dentro del sector agrícola y, a su vez, de la economía en general. Esto se debe principalmente al cambio tecnológico y al capital humano, que han sido mejorados mediante la práctica de políticas públicas acertadas para el sector cafetero, contrario a los sectores que no son significativos en el crecimiento, en los que se evidencia poca productividad y bajos rendimientos. Caso que se presenta con la industria manufacturera, que no genera un aporte significativo al PIB del departamento del Huila.

Resulta interesante, además, que el sector transporte sea determinante y generador de crecimiento en el departamento. Estudios realizados en Huila, como el Informe de Coyuntura Económica, realizado por la Cámara de Comercio del Huila en el año 2013, corroboran la importancia que tiene este sector a la hora de crear eslabonamientos (con otros sectores de vital importancia como la agroindustria) que mejoran y propenden por el crecimiento económico.

Por eso, el departamento está proporcionando además de proyectos en conectividad vial, avances en la línea férrea y plataforma de intercambio modal del norte del Cauca, interconexión vial al Pacífico con el puerto de Buenaventura, desarrollo vial en el sur del departamento del Meta, desarrollo del puerto de Tumaco, adecuación de la navegabilidad de los ríos Magdalena hasta Neiva, Putumayo y Caquetá, construcción de la vía férrea eco turística Puerto Nariño, Leticia, Tarapacá, corredor multimodal Tumaco, Puerto Asís, Belén, Topará, y variantes para el tráfico pesado en los municipios de Mocoa, Villa Garzón, Puerto Asís y Puerto Caicedo.

Se destaca en los planes de gobierno del departamento, el esfuerzo por hacer más competitivos los sectores productivos que lo componen. Sin embargo, la evidencia refleja ausencia de asociatividad en casi todas las actividades productivas exceptuando la caficultura que goza de una dinámica interesante desde adentro y hacia fuera, gracias a la organización que rodea la producción, caficultores asociados en cooperativas, organizaciones públicas y privadas estimulando y apoyando el sector, una Federación que brinda permanente apoyo técnico, vigilancia y control. El resultado, un sector que genera pujanza en el crecimiento de la región. Sin embargo, es un producto que internacionalmente no tiene un peso significativo en la balanza de pagos. 
En el Departamento del Huila, el principal renglón de la economía que aporta en gran medida al PIB Departamental es la minería (19\%), seguida por el sector agrícola incluyendo la participación del café (17\%), en el tercer lugar está el sector de construcción (11\%) por su dinamismo en los últimos años, el transporte con $8 \%$ ocupando el cuarto lugar, seguido por la industria (5\%) y por últimos el sector eléctrico (4\%).

\section{Referencias}

Ampié, R. L. (2000). Coherencia ecológica, condición para disminuir riesgos en el proceso de desarrollo rural sustentable de la agricultura. Recuperado de: http:// bibliotecavirtual.clacso.org.ar/ar/libros/rjave/mesa5/cerda.pdf

Barro, R. (mayo, 1991). Economic Growht in a Cross Section of Contries . Quarterly Journal of Economics.

Bernardo , J. y Caldero, J. F. (2000). Investigación Cuantitativa - Métodos no Experimentales. Madrid, España: RIAL S.A.

Bolsa Mercantil de Colombia [BMC $]$ (2011). Bolsa Mercantil de Colombia. Recuperado de: http://www.bna.com.co

Calzada, A. (marzo, 2002). El Impacto de la Inversión en el Crecimiento Económico de México de 1976 a 1996. Un Modelo Econométrico. México D.F: Universidad Nacional Autónoma de México, UNAM.

Centro Andino de Altos Estudios (febrero, 2007). DANE. Recuperado de https:// www.dane.gov.co/files/etnicos/cartilla_quibdo.doc

Clark, J. M. (diciembre, 1923). Studies in the Economics of Overhavied Cost. How and Why Large Plants Bring Economy. Chicago: University Of Chicago Press.

Departamento Administrativo Nacional de Estadísticas [DANE]. (septiembre, 2013). Informe de Coyuntura Económica Regional 2012. Recuperado de: http://www.dane.gov.co/files/icer/2012/huila_icer_12 
Fajardo, O. (febrero, 2013). La Minería y su Incidencia en el Crecimiento Económico del Departamento del Huila-Colombia. Revista Universitaria Digital de Ciencias Sociales, IV(6), 1-5. Recuperado de: www.cuautitlan. unam.mx/rudics - UNAM

Galor, O. (julio, 1996). Convergence? Inferences From Theoretical Models. Royal Economic Society, 106(437), 1056-1069. Recuperado de http://www. jstor.org/stable/2235378

Gobernación del Huila (2014). Anuarios Estadísticos Agropecuarios. Neiva, Colombia.

Harberger, A. C. (1983). The Cost-Benefit aproach to Development Economics, World Development. Oxford, England: Reprinted by permission from Elsevier Science Ltd.

Kalmanovitz, S., y López, E. (2006). La Agricultura en Colombia entre 1950 y 2000. En S. Kalmanovitz, y E. López, La agricultura en el siglo XX (pp. 197224). Bogotá: Borradores de Economía.

Lora, E. (2008). Técnicas de Medición Económica. Bogotá, Colombia: Alfaomega.

Lucas, R. (1988). On the Mechanics of Economic Development. Journal Of Monetary Economics, (22), 3-42. North-Holland: Elsevier Science Publishers.

Naciones Unidas [NU]; Comisión Economica Para América Latina y el Caribe Subsede México [CEPAL] (noviembre, 2003). Modelos Econométricos para los Paises de Centroamérica, (112).

Ríos, M. A. (agosto, 2014). Aspectos Teóricos del Crecimiento Econóomico. Recuperado de: http://www.eumed.net/libros-gratis/2005/mgr/1b.htm

Romer, D. (2006). EL Modelo de Crecimiento de Solow. Madrid, España: McGrawHill.

Romer, D. (2006). La Nueva Teoria del Crecimiento. La Naturaleza del Conocimiento y de los Factores Determinantes. Madrid, España: McGraw-Hill. 
Romer, P. M. (1986). Increasing Returns and Long-Run Growth. Journal of Political Economy, 94(5), 1002-1037. Recuperado de: http://www.jstor. org/stable/ 1833190

Ros, J. (2004). La Teoría del Desarrollo y la Economía del Crecimiento. Un Economía Madura: Crecimiento y Acumulación de Factores. México: FCE, CIDE.

Ross, R., y David, L. (septiembre, 1992). A Sensitivity Analysis of Cross-Country Growth Regressions. American Economic Association.

Solow, R. (febrero, 1970). A Contribution to Theory of Economic Growth. Quartely Journal of Economics.

Spanos, A. (octubre,1986). Statistical foundations of econometric modelling. Cambridge University Press.

Wooldridge, J. M. (2010). Introducción a la Econometría- Un Enfoque Moderno. Combinación de cortes transversales en el tiempo: métodos simples para datos de panel. México D.F: Cengage Learning. 